\title{
Performance-based pharmacy payment models: key components and critical implementation considerations for successful uptake and integration
}

\author{
Chloe Richard, MS; Benjamin Y Urick, PharmD, PhD; Shweta Pathak, MPH, PhD; John Jackson, BPharm, MPH; \\ and Melanie Livet, PhD
}

\section{What is already known about this subject}

- In the United States, there has been a recent shift toward value-based health care to improve patient outcomes while reducing health care spending.

- The development of performancebased pharmacy payment models (PBPPMs) has been led by commercial entities such as private insurance companies and pharmacy benefit managers.

- There is a lack of transparency in how these models are designed and implemented.

\section{What this study adds}

- This study identified 4 major components of PBPPMs as attribution, performance and quality measures, incentive structures, and patient care services.

- Recommendations to improve the design of PBPPMs include increased transparency of PBPPMs and increased alignment of incentive structure and performance measures to improve patient care.

- To facilitate the implementation of these models, it is important to plan and use roadmaps for successful uptake, build critical organizational infrastructure (eg, data sharing), and embrace a culture of quality and innovation across the health care spectrum.

\section{ABSTRACT}

BACKGROUND: In the United States, the shift towards value-based health care seeks to improve patient outcomes while reducing health care spending. Although the federal government has led the implementation of performance-based models for physicians and health care systems, commercial entities have largely been responsible for the development of similar models within pharmacy. For the purposes of this study, performancebased pharmacy payment models (PBPPMs) were defined as prescription drug payment models that determine reimbursement or fees for community pharmacies based in part on measured performance. These PBPPMs incentivize pharmacies to improve patient care by linking reimbursement to performance measures. However, the design and implementation of PBPPMs lack transparency and have not been described in the literature.

OBJECTIVES: To (1) describe the structure of PBPPMs in the United States and (2) identify contextual and motivational influences that

\begin{abstract}
Author affiliations
Chloe Richard, MS; Benjamin Y Urick, PharmD, PhD; Shweta Pathak, MPH, PhD; and Melanie Livet, PhD, Center for Medication Optimization (CMO), Division of Practice Advancement and Clinical Education, Eshelman School of Pharmacy, University of North Carolina at Chapel Hill. John Jackson, BPharm, MPH, Center for Medicine Use and Safety, Faculty of Pharmacy and Pharmaceutical Sciences, Monash University, Victoria, Australia.
\end{abstract}

\section{AUTHOR CORRESPONDENCE: \\ Chloe Richard, 716.969.7062; \\ chloe.richard@unc.edu}

J Manag Care Spec Pharm. 2021;27(11):1568-78

Copyright (C2021, Academy of Managed Care Pharmacy. All rights reserved.

need to be considered for successful uptake and integration of these models.

METHODS: A search of peer-reviewed and grey literature was undertaken. In addition, semi-structured stakeholder interviews were conducted with a convenience sample of 17 individuals who were community pharmacists, payers, quality measure developers and vendors, academics, and pharmacy advocacy organization leaders. Data were analyzed to understand the current structure of PBPPMs and opportunities for improvement, as well 
as implementation considerations that included facilitators, barriers, and key insights.

RESULTS: This study identified 4 major components of US PBPPMs: attribution, performance and quality measures, incentive structures, and patient care services. A number of barriers (eg, lack of alignment) and recommendations to improve the current structure of PBPPMs (eg, the need for adequate incentives to facilitate change) were highlighted. Notable implementation considerations centered around (1) establishing common ground among stakeholders to avoid misalignment and encourage engagement; (2) the importance of a quality-driven, innovative, and flexible organizational culture with access to data infrastructure, adjusted workflows, and relevant trainings; (3) supporting the cultural transition to value-based health care; and (4) application of financial incentives at the pharmacy or pharmacist level.

CONCLUSIONS: To better develop and implement PBPPMs, it is first critical to understand the key components that define these models and the needed changes to their structure. In addition, identifying the contextual and motivational factors that influence their successful integration can improve future uptake. This study illustrates the landscape of PBPPMs in the United States, as well as makes recommendations for improvement in their design. To improve future development and implementation of these models, the following recommendations are highlighted: (1) increase transparency and alignment of measures with the incentive structure; (2) embrace innovative business models; (3) carefully plan and use roadmaps that outline successful uptake and implementation; and (4) foster culture of quality at all levels of health care.

The shift towards value-based health care in the United Stated has resulted in payment models designed to improve patient care and outcomes while simultaneously decreasing the cost of care. ${ }^{1-3}$ Implemented by private insurers as well as by the federal government, these models adjust payments to incentivize health care systems and providers to improve performance on quality metrics and reduce health care spending. ${ }^{4,5}$ The federal government has led implementation of performance-based payment models primarily designed for physicians and health care systems through programs such as the Merit-based Incentive Payment System and the Medicare Shared Savings Program Accountable Care Organizations. ${ }^{6-8}$ Efforts to spearhead innovative performance-based pharmacy payment models (PBPPMs), on the other hand, have been led largely by commercial entities, such as private insurance companies and pharmacy benefit managers. As a result, the design and implementation of PBPPMs tend to lack the level of transparency typically observed with government-funded payment models.
Despite this shortcoming, use of PBPPMs to fund community pharmacies has expanded rapidly. The first prominent PBPPM was implemented by Inland Empire Health Plan in $2014 .{ }^{9}$ As of 2020 , quality is measured for $90 \%$ of Medicare beneficiaries, and $95 \%$ of pharmacies are connected to the Electronic Quality Improvement Platform for Plans and Pharmacies (EQuIPP), which curates these quality data. ${ }^{10}$ The potential for community pharmacies to engage with these models has been highlighted in the literature..$^{11-14}$

For pharmacies to benefit from using the payment mechanisms afforded by these models, and for payers to optimize their design, it is essential to first understand how they are structured, operated, and can best be implemented. Increased understanding of the components of PBPPMs and the context in which these models are used will facilitate their integration into practice..$^{15-18}$

The objectives of this study were to (1) describe PBPPMs as they are structured in the United States, including associated challenges and recommendations for improvements, and (2) identify the contextual and motivational influences that need to be considered when implementing these models. For the purposes of this study, PBPPMs were defined as prescription drug payment models that determine reimbursement or fees for community pharmacies based in part on measured performance.

Unlike traditional pharmacy payment models where pharmacies are paid a fixed fee for every prescription dispensed, with separate payment for patient care services when available, PBPPMs use performance measures to evaluate pharmacy performance and modify payments or fees. ${ }^{19,20}$ With performance-based funding, pharmacies are paid a bonus, withheld payment, or incur penalties based on measured performance, regardless of the service used to improve that metric.

\section{Methods}

\section{CONCEPTUAL FRAMEWORK}

The study aims were framed using implementation science best practices. This approach posits that effective integration of models and interventions depends on a thorough understanding of the innovation and its core components (ie, active features essential to achieve the intended outcomes); an awareness of the context in which this innovation is being deployed; and use of proactive implementation strategies. ${ }^{15-18}$ Gaining insights into the potential adoption and relevance of PBPPMs therefore requires that we first understand the current structure of PBPPMs and how they can be improved (Aim 1). This description involves a highlevel overview (Who is implementing PBPPMs? For whom? 


\section{FIGURE 1 Study Roadmap}
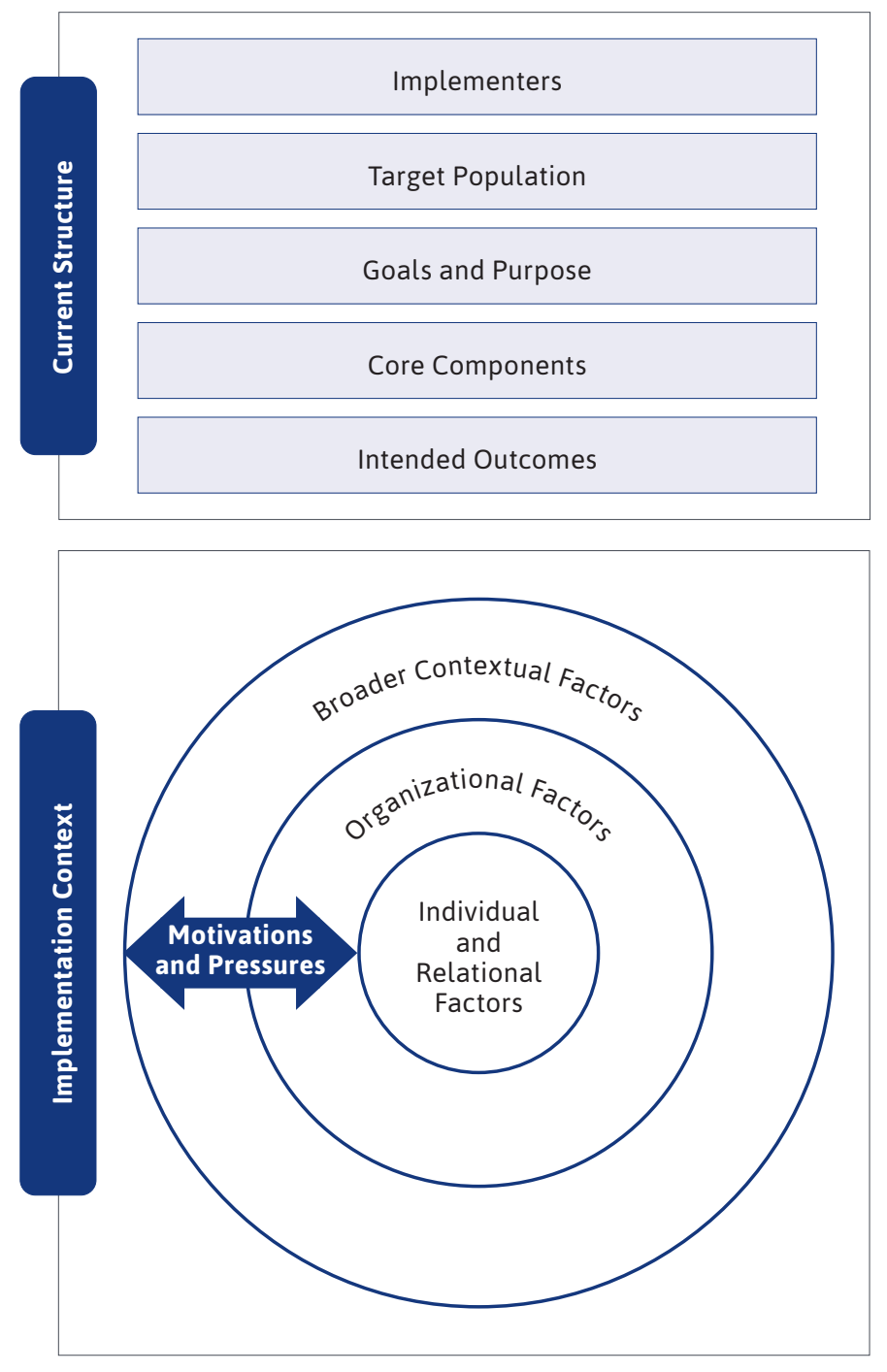

In what setting? For what purpose? To what end?) and an understanding of the PBPPM components.

Second, it is critical to establish the contextual conditions and motivational pressures of the environments in which these PBPPMs are deployed at multiple levels of the socioecological model (Aim 2): individual and relational (eg, pharmacists, payers, and patients); organizational (eg, pharmacies); and broader cultural context (eg, regulations). These implementation considerations, including facilitators, barriers, and key insights, are critical to facilitating successful uptake of these models in real-world environments. Figure 1 outlines the study organizing roadmap.

\section{STUDY DESIGN}

This exploratory qualitative study involved an environmental scan and semi-structured stakeholder interviews. Both methods were used to gain insights into PBPPMs, with the purpose being to seek convergence and strengthen the validity of findings through data triangulation. The environmental scan and stakeholder interviews in combination captured an overall description of PBPPMs, their primary components, and recommended modifications. In addition, both of these sources informed considerations for implementing PBPPMs, including facilitators, barriers, and lessons learned. The data abstracted from the environmental scan and interviews were analyzed separately and results from both were subsequently combined for synthesis and ease of reporting.

\section{DATA COLLECTION AND ANALYSIS}

The environmental scan (ie, search of existing peer-reviewed and grey literature) was conducted on PubMed and Google using the following terms: "value-based care," "pay-for-performance," or "performance-based" AND "pharmacy." The initial search uncovered 27 articles and documents. Review of the abstracts reduced the number of articles and documents to be extracted to 9 . These sources were examined for relevant information and extracted into a spreadsheet using a priori categories aligned with the study aims (eg, components of PBPPMS). Results were then synthesized to produce a summary document organized around the categories of interest. This summary was reviewed by the research team to ensure consensus on interpretation of the data within the broader context of PBPPMs.

The stakeholder interviews occurred via telephone over a 4-month period, with 17 stakeholders $(5$ pharmacists, 7 payers, 3 quality measure developers and vendors, 1 academic pharmacist, and 1 member of a national pharmacy organization). These interviewees represented a range of roles, from pharmacy owners to staff pharmacists and chief pharmacy officers to lower-level managed care pharmacists leading quality-related projects. The study team identified the initial set of eligible participants with snowball sampling used to identify additional candidates. This final sample size was determined to be appropriate based on sampling design recommendations..$^{21,22}$

The interview guide, created to standardize the discussions, inquired into the same domains of interest as the environmental scan. Specifically, interview questions focused on definition and essential features of PBPPMs, goals and impact of these models, implementation considerations, challenges, and recommendations for improvement. Informed consent was obtained before the interviews, and all participants were offered financial compensation 
for their time. Institutional review board approval was obtained before data collection.

Following completion of the interviews, interview transcripts were analyzed by the first author using content analysis. ${ }^{23}$ An a priori first-level coding structure was created based on the categories of interest (eg, payer). Following an initial reading of the transcripts to ensure familiarity with the material, the analyst applied first-level codes, while identifying second- and third-level codes (eg, performance and quality measures, alignment of measures). All transcripts were analyzed using the final codebook. This information was synthesized into a spreadsheet by code across all interviews.

To test inter-rater reliability, the third author also analyzed a 20\% sample of 3 interviews during the development of the coding structure. Cohen's Kappa demonstrated substantial agreement across both coders $(\kappa=0.76)$. Disagreements were discussed and resolved before further analysis. QSR International's NVivo 12 software was used to facilitate the coding process. ${ }^{24}$

\section{Results}

\section{DESCRIPTION OF PBPPMS: OVERVIEW, COMPONENTS,} AND RECOMMENDED CHANGES

Overview. PBPPM implementation occurs in different types of pharmacy settings (eg, independent pharmacies, chain pharmacies, and specialty pharmacies) by different entities (eg, pharmacists, health plans, pharmacy benefit managers, and government) and are used with Medicare, Medicaid, and commercial populations. The primary goals of these models were reported to be decreased total cost of health care spend and improved patient care. Secondary goals included optimizing the engagement of pharmacists with patients and incentivizing pharmacists to achieve better results on performance and quality measures. The actual impact of these models has been mixed, with reports of positive and negative outcomes. Lack of formal analysis of model impact makes assessing the extent to which models achieve these goals challenging.

Components of PBPPMs and Recommended Changes. Four primary components of PBPPMs emerged from the analysis: attribution, performance and quality measures, incentive structures, and patient care services. Table 1 contains a list of these components, along with associated challenges and recommended changes summarized from the environmental scan and interviews.

Attribution. The importance of attribution emerged in the context of attributing patients to pharmacies for quality measurement purposes and attributing impact to a pharmacy or pharmacist vs another provider. Current attribution models typically assign patients retrospectively based on prescription volume. Attribution models may be improved by attributing patients at point of care, although specific methods for doing so remain unclear. Attributing the impact of a specific pharmacy or pharmacist on a specific measure remains problematic because multiple providers can impact multiple quality measures. Delineating impact of a single provider can be challenging (eg, both pharmacists and providers could impact patient adherence).

Performance and quality measures. Performance and quality measures were identified as critical aspects of PBPPMs. These measures (examples from the environmental scan and interviews can be found in Table 2) were described as benchmarks to evaluate the impact of pharmacy services on patient outcomes. Results on these measures typically determine the performance-linked bonuses or penalties for participating pharmacies. The metrics incorporated into PBPPMs varied across services, payers, and contracts. Participants reported that measures typically assessed health care processes or intermediate outcomes (eg, medication adherence). They also tended to have been developed for other health care professions (eg, National Committee for Quality Assurance's Healthcare Effectiveness Data and Information Set (HEDIS) measures for medical providers) and payment settings (eg, Part D star ratings).

To address these challenges, it was suggested that PBPPMs include use of clinical and utilization outcomes metrics more closely tied to the results of care (eg, hospitalizations and total cost of care). To increase applicability to the care provided by pharmacies, current measures could either be adapted or new metrics could specifically be designed for use in community pharmacy (eg, medication sensitive hospitalizations). Finally, streamlining the number of measures included in PBPPMs and facilitating alignment across payers and lines of business (eg, commercial and Medicare) were identified as strategies to increase successful uptake by pharmacies.

Incentive structure. Traditionally, pharmacies generate revenue from dispensing activities with little financial incentive to provide enhanced patient care. PBPPMs incentivize pharmacies to perform on a given set of measures that are determined by the insurance plan, pharmacy benefit manager, or accountable care organization. Various mechanisms (eg, bonuses, penalties, withholds, adjustable reimbursement schedules, payment for improvement, payment for being at a certain percentile, payment for a specific service provided [fee for service], and quality 


\section{TABLE 1 Components of Performance-Based Pharmacy Payment Models in the United States}

\begin{tabular}{|c|c|c|}
\hline Model Components & Challenges & Recommendations \\
\hline Attribution & $\begin{array}{l}\text { - Attribution of patients to a specific pharmacy or } \\
\text { pharmacist } \\
\text { - Attribution of impact on a specific measure to a specific } \\
\text { pharmacy or pharmacist } \\
\text { - Separation of impact of one provider versus another on } \\
\text { a metric }\end{array}$ & - None provided \\
\hline $\begin{array}{l}\text { Performance and } \\
\text { quality measures }\end{array}$ & $\begin{array}{l}\text { - Lack of measure alignment across providers, payers, } \\
\text { contracts, and services } \\
\text { - Measures assess process instead of true outcomes } \\
\text { - Measures are less applicable to pharmacy because they } \\
\text { were created for other professions }\end{array}$ & $\begin{array}{l}\text { - Increase alignment of measures across pharmacist, } \\
\text { provider, payers, contracts, and services } \\
\text { - Utilize measures that are focused on tangible clinical } \\
\text { outcomes } \\
\text { - Utilize measures that are meaningful to pharmacy and } \\
\text { match pharmacists' skill sets }\end{array}$ \\
\hline Incentive structure & $\begin{array}{l}\text { - Lack of transparency in types of incentive structures } \\
\text { leads to uncertainty around expected incentives for the } \\
\text { pharmacy } \\
\text { - Incentive structures applied are often not optimal } \\
\text { (penalty over reward), and fee for service is still } \\
\text { predominantly used } \\
\text { - Incentives are focused on benchmarks and do not allow } \\
\text { for improvement within benchmarks } \\
\text { - Incentives are provided to pharmacies, and are not } \\
\text { passed down to pharmacists or patients } \\
\text { - Incentives are not adjusted for patients making progress } \\
\text { without reaching benchmarks } \\
\text { - Incentive structures can create disincentive to provide } \\
\text { appropriate patient care }\end{array}$ & $\begin{array}{l}\text { - Align incentives with (1) performance metrices that can } \\
\text { be impacted by pharmacy, (2) value of services, and } \\
\text { (3) patient outcomes } \\
\text { - Increased transparency of incentive structures within the } \\
\text { models } \\
\text { - Include large enough incentives to impact the type of } \\
\text { care provided } \\
\text { - Separate payment for dispensing medications and } \\
\text { incentives for patient care and improving metrics } \\
\text { - Align incentives across all stakeholders, including payers, } \\
\text { health care professionals, and patients }\end{array}$ \\
\hline Patient care services & $\begin{array}{l}\text { - Patients may not be receptive to pharmacists providing } \\
\text { services } \\
\text { - There is resistance from payers to pay for specific } \\
\text { patient care services } \\
\text { - There are regulatory barriers that prevent pharmacists } \\
\text { from being able to provide some services }\end{array}$ & $\begin{array}{l}\text { - There should be continued evolvement toward } \\
\text { pharmacists improving medication use metrics (opposed } \\
\text { to dispensing metrics) } \\
\text { - Pharmacists should have the opportunity to provide } \\
\text { patient care as they are seen as valuable and accessible } \\
\text { providers with the ability to engage patients } \\
\text { - Payers should not be prescriptive in the patient services } \\
\text { pharmacists need to provide }\end{array}$ \\
\hline
\end{tabular}

grants) modify dispensing revenue instead of providing separate bonuses.

Recommended changes included (1) increased transparency with the incentive structure to facilitate community pharmacists' understanding of the performance models in which they are involved and reduce uncertainties around what pharmacies ultimately receive; (2) greater incentive alignment across all stakeholders, as well as between performance goals and patient metrics, to reduce the need to choose between competing priorities such as meeting a performance goal or providing optimal patient care (eg, providing 90-day fills to patients to meet adherence metrics when 30-day fills to increase patient touchpoints may be preferable for patient care); and (3) appropriately size and scope financial rewards to support the type of broad-based practice change needed to maintain financial viability, while delivering better patient care within a value-based environment. Participating pharmacies should be recognized for improving patient care and making progress towards benchmarks, rather than solely achieving metric benchmarks.

Patient care services. An essential aspect of PBPPMs was delivery of patient care services identified as having potential to improve patient outcomes (eg, comprehensive medication reviews, medication therapy management, medication synchronization, and immunizations). Overall, participants reported that the delivery of patient care services had a beneficial impact on patient care, although 


\section{TABLE 2 Examples of Performance and Quality Measures from Environmental Scan and Interviews}

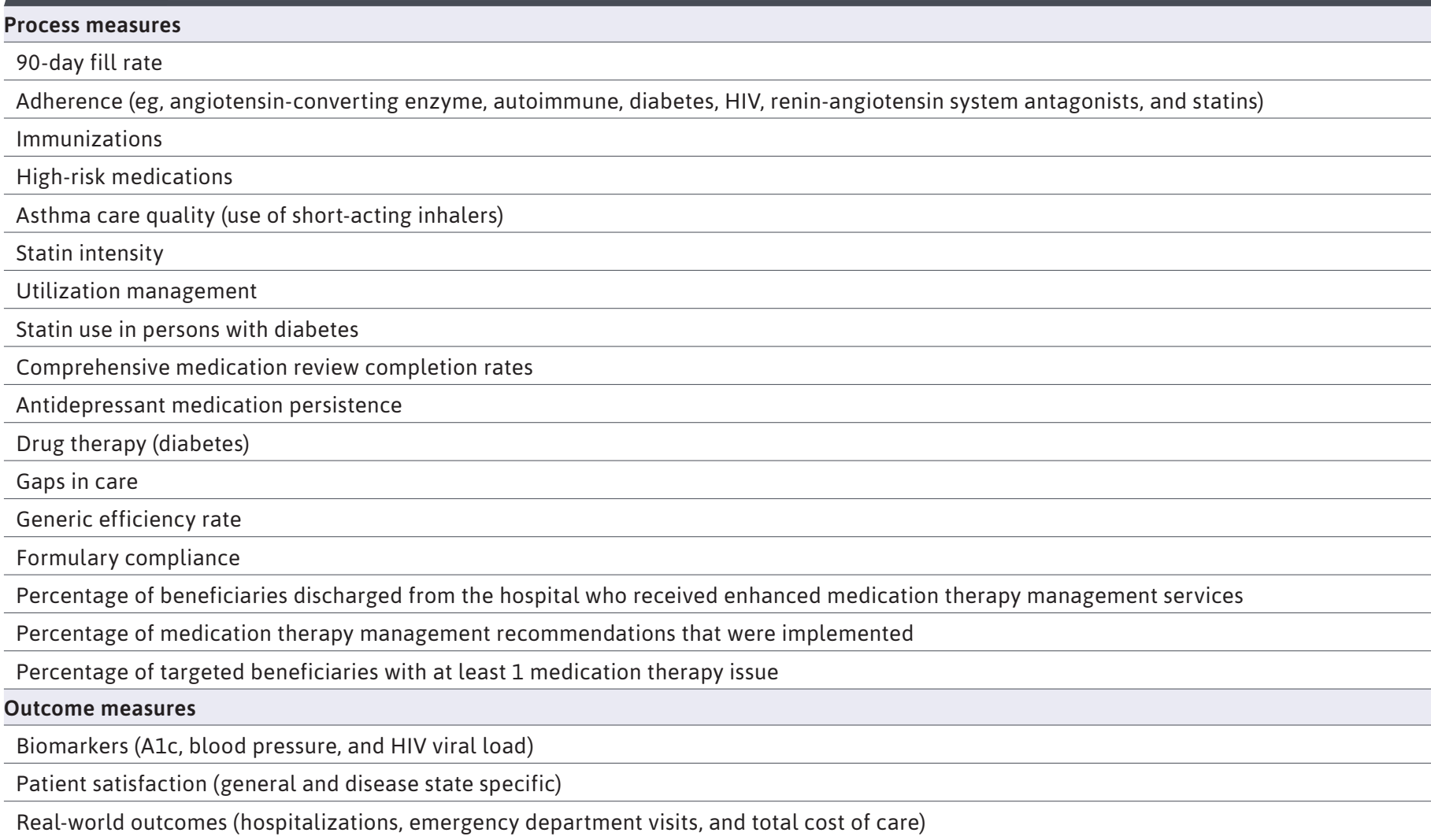

payers expressed resistance to paying for these services outside of PBPPMs. It was also noted that some patients may be hesitant to use these services.

There was an identified desire to have pharmacists improve medication use metrics (eg, impact on hemoglobin A1c or blood pressure) rather than focus on standard medication dispensing metrics (eg, prescription volume). However, to achieve success, pharmacists need to overcome regulatory barriers that impede expanded scope of practice to provide specific patient care services (eg, point of care testing). Ultimately, it was recommended that pharmacies be able to select the most appropriate services to improve the health of their patient population and impact metrics.

\section{IMPLEMENTATION CONSIDERATIONS: CONTEXTUAL CONDITIONS AND MOTIVATIONAL PRESSURES}

Implementation considerations including specific factors, key barriers and facilitators, and recommendations summarized from the environmental scan and interviews can be found in Table 3.
Individual and Relational Factors. By definition, PBPPMs require involvement of multiple stakeholders, including pharmacists and other health care professionals, payers, and patients. These groups often have conflicting interests and viewpoints, which leads to overly complex models, and ultimately hinders the uptake and success of PBPPMs. Recommendations to address these barriers included aligning interests by identifying common ground across stakeholder groups; enhancing clarity around PBPPMs; driving performance by creating incentives for collaborative care (eg, pharmacists and physicians); gathering buy-in from all stakeholder groups to improve current PBPPMs; identifying engaged leadership and champions who can facilitate uptake of PBPPMs; and ensuring that pharmacies are effectively engaging with their patients.

Organizational Characteristics. A pharmacy's organizational culture and infrastructure were identified as 2 important aspects of the implementing environment that deserved consideration. The pharmacy organizations that have found most success with these models seemed to have 


\section{TABLE 3 Implementation Considerations of Performance-Based Pharmacy Payment Models}

\begin{tabular}{|c|c|c|c|}
\hline $\begin{array}{c}\text { Implementation } \\
\text { influences }\end{array}$ & Barriers & Facilitators & Recommendations \\
\hline \multicolumn{4}{|c|}{ Individual and relational factors } \\
\hline & $\begin{array}{l}\text { - There can be conflicting interests } \\
\text { across stakeholder groups } \\
\text { - Maintaining buy-in and engagement } \\
\text { of pharmacists and patients }\end{array}$ & & $\begin{array}{l}\text { - Establish positive relationships } \\
\text { between stakeholders and identify } \\
\text { common ground } \\
\text { - Create incentives for collaborative } \\
\text { care } \\
\text { - Obtain buy-in from all stakeholder } \\
\text { groups }\end{array}$ \\
\hline \multicolumn{4}{|c|}{ Organizational characteristics } \\
\hline Culture & & $\begin{array}{l}\text { - Overall culture of engagement, } \\
\text { flexibility, and innovation } \\
\text { - Individuals within successful } \\
\text { organizations are creative, energized, } \\
\text { and self-motivated }\end{array}$ & $\begin{array}{l}\text { - Pharmacists and payers need to } \\
\text { be engaged, and drive patient } \\
\text { engagement } \\
\text { - There needs to be a willingness to } \\
\text { change at a bold, innovative pace }\end{array}$ \\
\hline \multicolumn{4}{|c|}{ Organizational infrastructure } \\
\hline $\begin{array}{l}\text { Information } \\
\text { technology }\end{array}$ & $\begin{array}{l}\text { - The vast number of sources for } \\
\text { performance and patient data is } \\
\text { overwhelming } \\
\text { - Performance data is not always } \\
\text { readily shared with pharmacies or } \\
\text { pharmacists } \\
\text { - Pharmacists are sometimes unaware } \\
\text { of performance data } \\
\text { - There can be a lack of infrastructure } \\
\text { for sharing clinical and performance } \\
\text { data }\end{array}$ & $\begin{array}{l}\text { - Having a platform or other way to } \\
\text { share quality metrics information } \\
\text { within and across pharmacies } \\
\text { - Having an internal system to share } \\
\text { data } \\
\text { - Having clear reports that outline } \\
\text { performance and money lost or } \\
\text { gained }\end{array}$ & $\begin{array}{l}\text { - Increase access to and training on } \\
\text { EQuIPP } \\
\text { - Integrate electronic health record, } \\
\text { dispensing, and performance } \\
\text { measurement into one system } \\
\text { - Allow pharmacists to bill for services }\end{array}$ \\
\hline $\begin{array}{l}\text { Workflow } \\
\text { operations }\end{array}$ & $\begin{array}{l}\text { - Already existing high workloads } \\
\text { - Lack of incentive to change workflow } \\
\text { - Lack of time and space } \\
\text { - Differing workflows necessitated by } \\
\text { the variety of models which increases } \\
\text { administrated burden }\end{array}$ & $\begin{array}{l}\text { - Optimizing the use of the pharmacist } \\
\text { by: } \\
\text { - use of technicians } \\
\text { - use of central fill } \\
\text { - automation } \\
\text { - use of care managers }\end{array}$ & \\
\hline Training & $\begin{array}{l}\text { - Pharmacists are sometimes unaware } \\
\text { they are in a performance-based } \\
\text { program and do not understand } \\
\text { performance measures or their } \\
\text { connection to payment } \\
\text { - Nonpharmacy providers are unaware } \\
\text { pharmacists are being measures on } \\
\text { performance measures }\end{array}$ & & $\begin{array}{l}\text { - Training and education should be } \\
\text { provided on performance and quality } \\
\text { measures and address: } \\
\text { - reasons for using the measures } \\
\text { - relevant metrics } \\
\text { - platforms (eg, EQuIPP) } \\
\text { - relevant clinical interventions }\end{array}$ \\
\hline \multicolumn{4}{|c|}{ Broader contextual influences } \\
\hline & $\begin{array}{l}\text { - There is some resistance to shift } \\
\text { toward the pay for outcomes-based } \\
\text { programs compared to continuing } \\
\text { with the more traditional pharmacy } \\
\text { role } \\
\text { - Lack of recognition of pharmacists } \\
\text { as health care providers and } \\
\text { reimbursement for all pharmacist } \\
\text { provided services }\end{array}$ & $\begin{array}{l}\text { - Current regulatory environment } \\
\text { allows opportunities for providers } \\
\text { and pharmacists to work together to } \\
\text { improve performance }\end{array}$ & $\begin{array}{l}\text { - Bold and innovative change } \\
\text { - Continual cultural shift that supports } \\
\text { pharmacist reimbursement } \\
\text { - Involving payers and pharmacists to } \\
\text { engage patients } \\
\text { - Pharmacies and pharmacists should } \\
\text { shift away from primarily engaging } \\
\text { in dispensing activities and embrace } \\
\text { provision of patient care services }\end{array}$ \\
\hline
\end{tabular}




\section{TABLE 3 Implementation Considerations of Performance-Based Pharmacy Payment Models (continued)}

\begin{tabular}{|c|c|c|c|}
\hline $\begin{array}{c}\text { Implementation } \\
\text { influences }\end{array}$ & Barriers & Facilitators & Recommendations \\
\hline \multicolumn{4}{|c|}{ Motivations and pressures } \\
\hline & $\begin{array}{l}\text { - Lack of direct incentives applied to } \\
\text { individual pharmacists } \\
\text { - Increased pressure to provide } \\
\text { additional patient care } \\
\text { - Lack of self-motivation, time, and } \\
\text { confidence to provide patient care }\end{array}$ & $\begin{array}{l}\text { - Receiving direct individual financial } \\
\text { incentives } \\
\text { - Seeing need for pharmacies to } \\
\text { recognize their current business } \\
\text { model as unsustainable and need } \\
\text { to shift toward clinical services as } \\
\text { source of revenue } \\
\text { - Pharmacists' desire to practice at the } \\
\text { top of their license } \\
\text { - Professional satisfaction } \\
\text { - Desire to provide enhance patient } \\
\text { care } \\
\text { - Pharmacists' ability to see the impact } \\
\text { of their work }\end{array}$ & $\begin{array}{l}\text { - Build the confidence of community } \\
\text { pharmacists to provide patient care } \\
\text { services } \\
\text { - Apply financial incentives at } \\
\text { individual pharmacy or pharmacist } \\
\text { level }\end{array}$ \\
\hline
\end{tabular}

EQuIPP = Electronic Quality Improvement Platform for Plans and Pharmacies.

established a culture of engagement, flexibility, and innovation. Individuals within these organizations were identified as creative, energized, self-motivated, and empowered to select what they considered to be the most suitable intervention to improve metrics.

In addition to a culture embracing change, the use of PBPPMs was facilitated if a pharmacy had the following infrastructure elements in place: (1) information technology (IT)-enabled data capabilities to document and share metrics within and across pharmacies and allow individual pharmacies to understand their performance and incentives lost or gained; (2) supportive workflow operations that clearly outlined roles and responsibilities and allowed time for the patient care service to be delivered; and (3) training processes to ensure that members of the pharmacy team understood performance metrics, reasons for using them, platform options, and implications for reimbursement.

Recommended operational improvements included efforts to build an integrated IT data infrastructure across diverse PBPPMs to facilitate use (eg, clear performance reports) and billing. Also highlighted was the need to optimize the required shifts in workflows by streamlining expectations across PBPPMs (ie, each model requires a different workflow) and capitalizing on pharmacist capabilities with patient care services delivery (eg, by using technicians to drive dispensing, central fill, automation, and care managers to support pharmacy services).
Broader Contextual Influences. Successful implementation of PBPPMs seems to be predicated in part on a health care business culture focused on incentivizing quality-related patient care services. To move this culture forward, bold and innovative rather than incremental changes are required. As the broader health care environment shifts towards performance-based payment models, results highlighted the need for community pharmacies to recognize that their current business model is unsustainable and the need to shift into delivery of clinical services as a source of future revenue to offset reduced dispensing-related reimbursement.

Motivations and Pressures. The analysis identified many factors that improve and hinder motivation to actively engage in PBPPMs. Pharmacists who are most motivated to use these models were described as having a desire to practice at the top of their license. In addition, they tended to be professionally satisfied when afforded the opportunity to provide patient care and experience the tangible results of their work. Unfortunately, even motivated pharmacists experience challenges that dampen their enthusiasm, including lack of individual financial rewards and additional pressures associated with delivery of patient care services (eg, lack of time and confidence to deliver patient care services). To motivate pharmacist engagement with PBPPMs, applying financial incentives at the pharmacy or pharmacist level was recommended. 


\section{Discussion}

The shift towards value-based health care in the United States has led to experimentation with performance-based payment models. These models determine payments to providers and entities based in part on measured performance. ${ }^{20}$ These models have been used with physicians and hospitals, but less often to reimburse for services in community pharmacies. ${ }^{6-8}$ Because the move towards performance-based payment models in the United States seems inevitable, pharmacy payment models must evolve to facilitate the successful transition of community pharmacies to a value-based environment. To ensure uptake and use of PBPPMs, it is essential to first understand their design and implementation. To our knowledge, this is the first study to date that describes PBPPMs as they are currently structured and identifies the contextual conditions and motivational pressures that need to be considered for successful uptake.

This study uncovered 4 key components of PBPPMs: attribution, performance and quality measures, incentive structures, and patient care services. In addition, findings highlighted critical implementation considerations to facilitate uptake at multiple levels of the health care system, including individual and relational factors, organizational factors, broader contextual factors, and other motivations and pressures. The analysis revealed key insights that can be used to improve the design of PBPPMs and create an implementation environment to facilitate their use.

First and foremost, there seemed to be a high level of variability within and across PBPPMs. A variety of payers have implemented these models, and there is substantial variation in purpose, performance measures, and incentive structures. Performance metric thresholds are sometimes too high for pharmacies to meet, resulting in reduced payment. Additionally, incentives associated with current performance measures may not align well with patient care goals, which creates conflict between providing optimal patient care and increasing revenue. This variation and misalignment introduce uncertainty around pharmacy reimbursement. Greater transparency and alignment of measures, as well as incentives to improve quality of care, are recommended to facilitate effective uptake of PBPPMs.

Second, despite the challenges faced by community pharmacists transitioning to offering patient care, the value of this expanded role is recognized. Pharmacists are regarded as readily accessible to patients, ${ }^{25}$ well trusted ${ }^{26}$ and providing safe and effective dispensing of medications. As use of PBPPMs expands, there seems to be a desire for pharmacists to improve medication use metrics beyond traditional dispensing metrics. This may be facilitated by the development of models that separate payment for dispensing medications from incentives for improved patient care. Pharmacists are encouraged to embrace innovative business models to thrive in a value-based environment.

Third, misaligned interests across implementers can result in overly complex models that reduce pharmacist engagement with PBPPMs. Pharmacists may struggle with the complexities of the models or even be unaware they are in a performance-based program. This aligns with a recent survey of community pharmacy owners that found substantial frustration with PBPPMs as currently implemented. ${ }^{13}$ The multitude of stakeholders involved in delivery of these models have conflicting expectations that need to be addressed and resolved. It is recommended that input and feedback be gathered, reviewed, and synthesized to create the next generation of PBPPMs.

Fourth, effective implementation of PBPPMs was found to require use of data platforms and processes to facilitate data sharing; the capacity to successfully navigate workflow adjustments; and trainings to support pharmacist awareness and understanding of PBPPMs. These findings align with previous literature identifying the organizational infrastructure necessary to drive change in pharmacy business practices and adoption of innovative reimbursement pathways..$^{14,27,28}$ Uptake of PBPPMs will be facilitated through careful planning and use of an implementation roadmap that includes the essential operational components for successful integration.

Finally, there was a recognition that the successful transition to PBPPMs could be facilitated by a broader cultural shift supporting this evolution in community pharmacy. The importance of a culture of quality was highlighted in this study at the individual, organizational, health care system, and regulatory levels. To support the engagement of pharmacies within the value-based health care landscape, it is recommended that a culture of quality is fostered across the health care spectrum through continued collaboration between payers, pharmacies, pharmacists, and other health care providers, as well as the professional organizations that represent their interests.

\section{LIMITATIONS}

While this study provides many fruitful insights, it is also important to consider its limitations. First, as an exploratory study, it did not assess outcomes or effectiveness of PBPPMs; rather, it aimed to understand the components of these models and the contextual and motivational influences to be considered for successful integration. Second, this study has limited generalizability due to sample size within the layers of the socioecological model and recruitment strategy. In addition, the pharmacist interviewees 
represented independent and regional chain pharmacies rather than retail chain pharmacies. Finally, the analysis did not differentiate among the perspectives of stakeholder views. Additional analyses at each level could lead to insights into potential differences across stakeholder groups.

\section{Conclusions}

This study illustrates the landscape of PBPPMs in the United States. Recommendations to improve the design of PBPPMs and facilitate their uptake include improved transparency and alignment of measures with the incentive structure; embracing innovative business models; careful planning and use of roadmaps that outline successful uptake and implementation; and fostering a culture of quality at all levels of health care.

Future research should focus on exploring the commonalities and differences in perspectives across stakeholder groups; supporting effective designs of PBPPMs; further examining implementation facilitators and barriers under different conditions (eg, types of organizations); and investigating the effectiveness of these models on financial and patient care outcomes. Ultimately, inclusive, well-designed models and use of active strategies to facilitate implementation will drive the successful uptake of PBPPMs.

\section{DISCLOSURES}

This study was sponsored by PharmAlliance, an alliance between the pharmacy schools of the University of North Carolina at Chapel Hill, Monash University, and University College London. Urick reports consulting fees from Pharmacy Quality Solutions and Cardinal Health, unrelated to this work. The other authors have nothing to disclose.
This study was a podium presentation under the title "What Makes PerformanceBased Pharmacy Payment Models Work?" at AMCP Nexus Virtual, October 2020.

\section{REFERENCES}

1. Porter ME. What is value in health care? N Engl J Med. 2010;363(26):2477-81.

2. Wilson H, Gole J, Mishra B, Mishra J. Value based healthcare. Adv Manag. 2016;9(1):1-8.

3. Health Care Payment Learning and Action Network. APM measurement: progress of alternative payment models. 2019 methodology and results report. 2019. Accessed August 19, 2020. http:// hcp-lan.org/workproducts/apm-methodology-2019.pdf

4. Centers for Medicare \& Medicaid Services. Quality payment program. 2018. Accessed May 22, 2020. https://qpp.cms. gov/

5. Conrad DA. The theory of value-based payment incentives and their application to health care. Health Serv Res. 2015;50:2057-89.

6. Mendelson A, Kondo K, Damberg C, et al. The effects of pay-for-performance programs on health, health care use, and processes of care: a systematic review. Ann Intern Med. 2017;166(5):341-53.

7. Kondo KK, Damberg CL, Mendelson A, et al. Implementation processes and pay for performance in healthcare: a systematic review. J Gen Intern Med. 2016;31(1):61-69.

8. Vlaanderen FP, Tanke MA, Bloem BR, et al. Design and effects of outcomebased payment models in healthcare: a systematic review. Eur J Heal Econ. 2019;20(2):217-32.

9. Inland Empire Health Plan. Inland Empire Health Plan. 2020. Accessed February 26, 2020. http://www.iehp.org 10. Pharmacy Quality Solutions. Website. 2020. Accessed May 22, 2020. https:// www.pharmacyquality.com/
11. Pringle JL, Rucker L, Domann D, Charn C, Tice B, Burns AL. Applying value-based incentive models within community pharmacy practice. Pharmacy Times. February 16, 2016. Accessed September 9, 2021. https:// www.pharmacytimes.com/view/ applying-valuebased-incentive-modelswithin-community-pharmacy-practice

12. Avalere Health. Developing trends in delivery and reimbursement of pharmacist services. November 2015. Accessed February 26, 2020. https://www.copharm. org/assets/docs/103015 Avalere NACDS WhitePaper LP Final.pdf

13. Urick BY, Pathak S, Hughes TD, Ferreri SP. Design and effect of performance-based pharmacy payment models. J Manag Care Spec Pharm. 2021;27(3): 306-15. doi: 10.18553/jmcp.2021.27.3.306

14. Newman TV, Hernandez I, Keyser D, et al. Optimizing the role of community pharmacists in managing the health of populations: barriers, facilitators, and policy recommendations. J Manag Care Spec Pharm. 2019;25(9):995-1000. doi:10.18553/ jmcp.2019.25.9.995

15. Brownson R, Colditz G, Proctor E, eds. Dissemination and Implementation Research in Health: Translating Science to Practice. 1st ed. Oxford University Press; 2012.

16. Ogden T, Fixsen DL. Implementation science: a brief overview and a look ahead. Z Psychol. 2014;222(1):4-11. doi:10.1027/2151-2604/a000160

17. Livet M, Haines ST, Curran GM, et al. Implementation science to advance care delivery: a primer for pharmacists and other health professionals. Pharmacotherapy. 2018;38(5):490-502. doi:10.1002/phar.2114

18. Eccles MP, Mittman BS. Welcome to implementation science. Implement Sci. 2006;1(1):1. doi:10.1186/1748-5908-1-1

19. Jackson J, Urick B. Pay pharmacists to improve our health, not just supply medicines. The Conversation. October 10, 2019. Accessed February 26, 2020.. https://theconversation.com/ pay-pharmacists-to-improve-our-healthnot-just-supply-medicines-124641. 
20. Jackson J, Urick B. Performance-based pharmacy payment models: the case for change. Aust Heal Rev. 2019;43(5):502-07.

21. Malterud K, Siersma VD, Guassora AD. Sample size in qualitative interview studies: guided by information power. Qual Health Res. 2016;26(13):1753-60.

22. Guest G, Bunce A, Johnson L. How many interviews are enough? An experiment with data saturation and variability. Field Methods. 2006;18(1):59-82. doi: 10.1177/1525822X05279903

23. Hsieh HF, Shannon SE. Three approaches to qualitative content analysis. Qual Health Res. 2005;15(9):1277-88. doi:10.1177/1049732305276687
24. QSR International. NVivo Qualitative Data Analysis Software. Version 12. 2018. Accessed February 26, 2020. https:// www.qsrinternational.com/nvivoqualitative-data-analysis-software/ home

25. Tsuyuki RT, Beahm NP, Okada H, Al Hamarneh YN. Pharmacists as accessible primary health care providers: review of the evidence. Can Pharm J (Ott). 2018;151(1):4-5.

26. Marotta R. Pharmacists remain among most trusted and ethical professionals. Pharmacy Times. February 9, 2018. Accessed June 26, 2018. https:// www.pharmacytimes.com/news/ pharmacists-remain-among-mosttrusted-and-ethical-professionals
27. Goode JV, Owen J, Page A, Gatewood S. Community-based pharmacy practice innovation and the role of the community-based pharmacist practitioner in the United States. Pharmacy (Basel). 2019;7(3):106.

28. Reyes LD, Hong J, Lin C, Hamper J, Kroon L. Community pharmacists' motivation and barriers to providing and billing patient care services. Pharmacy (Basel). 2020;8(3):145. 